\title{
Low mass dimuon production in proton-nucleus and Indium-Indium collisions
}

\section{Hermine K. Wöhri* for the NA60 Collaboration}

CFTP, Instituto Superior Técnico, Lisbon, Portugal

E-mail: Hermine.Woehri@cern.ch

R. Arnaldi ${ }^{10}$, R. Averbeck ${ }^{9}$, K. Banicz ${ }^{2,4}$, J. Castor $^{3}$, B. Chaurand ${ }^{7}$, C. Cicalò ${ }^{1}$, A. Colla ${ }^{10}$, P. Cortese ${ }^{10}$, S. Damjanovic ${ }^{4}$, A. David ${ }^{2,5}$, A. De Falco ${ }^{1}$, A. Devaux ${ }^{3}$, A. Drees ${ }^{9}$, L. Ducroux ${ }^{6}$, H. En'yo ${ }^{8}$, A. Ferretti ${ }^{10}$, M. Floris ${ }^{1}$, P. Force ${ }^{3}$, A. Förster ${ }^{2}$, N. Guettet ${ }^{2,3}$, A. Guichard ${ }^{6}$, H. Gulkanian ${ }^{11}$, J. Heuser ${ }^{8}$, M. Keil ${ }^{2,5}$, L. Kluberg ${ }^{2,7}$, C. Lourenço ${ }^{2}$, J. Lozano ${ }^{5}$, F. Manso ${ }^{3}$, A. Masoni ${ }^{1}$, P. Martins ${ }^{2,5}$, A. Neves ${ }^{5}$, H. Ohnishi ${ }^{8}$, C. Oppedisano ${ }^{10}$, P. Parracho ${ }^{2}$, P. Pillot ${ }^{6}$, G. Puddu ${ }^{1}$, E. Radermacher ${ }^{2}$, P. Ramalhete ${ }^{2}$, P. Rosinsky ${ }^{2}$, E. Scomparin ${ }^{10}$, J. Seixas ${ }^{2,5}$, S. Serci ${ }^{1}$, R. Shahoyan ${ }^{2,5}$, P. Sonderegger ${ }^{5}$, H.J. Specht ${ }^{4}$, R. Tieulent ${ }^{6}$, G. Usai ${ }^{1}$, R. Veenhof ${ }^{2,5}$

${ }^{1}$ Univ. di Cagliari and INFN, Cagliari, Italy; ${ }^{2}$ CERN, Geneva, Switzerland; ${ }^{3}$ LPC, Univ. Blaise Pascal and CNRS-IN2P3, Clermont-Ferrand, France; ${ }^{4}$ Univ. Heidelberg, Heidelberg, Germany;

${ }^{5}$ Instituto Superior Técnico, Lisbon, Portugal; ${ }^{6}$ IPN-Lyon, Univ. Claude Bernard Lyon-I and CNRS-IN2P3, Lyon, France; ${ }^{7}$ LLR, Ecole Polytechnique and CNRS-IN2P3, Palaiseau, France; ${ }^{8}$ RIKEN, Wako, Saitama, Japan; ${ }^{9}$ SUNY Stony Brook, New York, USA; ${ }^{10}$ Univ. di Torino and INFN, Italy; ${ }^{11}$ YerPhI, Yerevan, Armenia

The NA60 experiment studied low-mass dimuon production in proton-nucleus and indium-indium collisions at the CERN SPS. While the p-A data can be well described by a superposition of light neutral meson decays, an excess is observed in the heavy-ion data, increasing with collision centrality, with respect to these expected sources. Thanks to the high statistics and good quality of our measurement, we can subtract the muon pairs due to $\eta, \eta^{\prime}, \omega$ and $\phi$ decays from the signal dimuon mass distribution. The resulting spectra are compatible with a $\rho$ spectral function broadening from peripheral to central collisions, with no change in the pole mass.

International Europhysics Conference on High Energy Physics

July 21st - 27th 2005

Lisboa, Portugal

\footnotetext{
* Speaker.
} 
QCD matter at the extreme temperatures and baryon densities provided by heavy-ion collisions is being studied at the CERN SPS since 1986. The CERES experiment, in particular, showed that the production of low-mass electron pairs in p-Be collisions could be explained on the basis of electromagnetic decays of the light neutral mesons alone [1], while in $\mathrm{S}-\mathrm{Au}$ and $\mathrm{Pb}-\mathrm{Au}$ [2] collisions an excess of a factor of 2-3 was observed in the mass range $200-700 \mathrm{MeV}$. This excess was interpreted as signaling in-medium modifications of the $\rho$ meson, which could indicate that chiral symmetry was partially restored. The poor statistical accuracy and mass resolution of the data, however, severely limited the understanding of the physics mechanisms behind the measured data. To clarify these and other observations, NA60 measured low mass dimuon production in proton-nucleus and in indium-indium collisions, at SPS energies, with good statistics, dimuon mass resolution and signal/background ratio.

In 2002 NA60 collected data with a $400 \mathrm{GeV}$ proton beam and three different nuclear targets simultaneously placed along the beam axis: $\mathrm{Be}$, In and $\mathrm{Pb}$. The muon spectrometer was complemented by a silicon micro-strip vertex tracker inside a $2.5 \mathrm{~T}$ dipole magnet, which provided the kinematics of the reconstructed charged tracks and, by fitting their common origin, the vertex of the primary interaction. This allowed us to separate the measured dimuons in three different samples, depending on the target where the interaction took place. Matching of the identified muons to suitable tracks reconstructed in the vertex tracker, in angles and momentum, significantly improved the dimuon mass resolution and reduced the level of combinatorial background from $\pi$ / K decays from $30 \%$ to $10 \%$. Figure 1 shows the mass distribution of the dimuons produced in $\mathrm{p}$-Be collisions and reconstructed in the apparatus, within the phase space window $3.3<y_{\text {lab }}^{\mu \mu}<4.2,\left|\cos \theta_{\mathrm{CS}}\right|<0.5$, $\eta_{\mu}<4.2, m_{\mathrm{T}}>0.7 \cdot\left(y_{\mathrm{lab}}-4.2\right)^{2}+0.4 \mathrm{GeV}$, where $\theta_{\mathrm{CS}}$ is the Collins-Soper angle. In this window the $\omega$ and $\phi$ mesons have acceptances 3.2-4.0\% and 6.1-7.2\%, respectively, depending on the $z$ position (sub-target) where they were produced. Their differential acceptances do not vary by more than a factor of 10 between any two selected events [3].

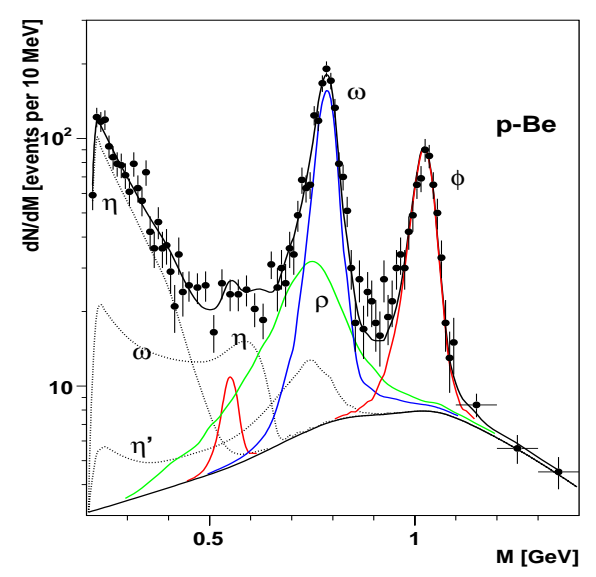

\begin{tabular}{cccc}
\hline & $\sigma^{\omega}[\mathrm{mb}]$ & $\sigma^{\phi}[\mathrm{mb}]$ & $\sigma^{\phi} / \sigma^{\omega}$ \\
\hline $\mathrm{Be}$ & $47.6 \pm 1.4$ & $3.0 \pm 0.1$ & $0.062 \pm 0.003$ \\
$\mathrm{In}$ & $439 \pm 14$ & $32.3 \pm 1.6$ & $0.074 \pm 0.004$ \\
$\mathrm{~Pb}$ & $571 \pm 22$ & $49.8 \pm 2.5$ & $0.087 \pm 0.006$ \\
\hline
\end{tabular}

Figure 1: Left: Fit to the dimuon mass spectrum collected in p-Be interactions. Right: $\omega$ and $\phi$ full phase space production cross-sections, and their ratio, for each p-A system (statistical error bars only).

The measured distributions are well described by the superposition of expected light hadron decays. From independent fits to the three dimuon mass distributions, imposing $\rho / \omega=1.1$, we extracted the $\omega$ and $\phi$ production cross-sections given in Fig. 1, after accounting for acceptances, efficiencies and luminosity. They were extrapolated to full phase space using kinematical distribu- 
tions [3] which we cannot check outside of our restricted window, including a uniform decay angle distribution for the 2-body decays. Systematical errors due to luminosities, efficiencies, etc, are negligible in comparison to the uncertainties related to this extrapolation, roughly estimated to be around $20 \%$. The $\sigma^{\phi} / \sigma^{\omega}$ cross-section ratio is essentially independent of the assumed kinematical distributions, and has a systematical uncertainty around $5 \%$.

For the 2003 heavy-ion run the vertex tracker was rebuilt with 12 tracking stations of radiation tolerant silicon pixel detectors (800000 pixels). Figure 2-left shows the measured opposite-sign dimuon mass distributions, the backgrounds and the extracted signal, integrated over all collision centralities; 360000 signal events, corresponding to around $50 \%$ of the collected statistics. The combinatorial background from $\pi$ and $\mathrm{K}$ decays was evaluated using a mixed-event technique [4], in $\sim 6000$ sub-samples such that different event topologies (event multiplicity, acceptance variations due to interactions at different $z$ position or different detector setups) are correctly accounted for; the accuracy reached is about $1 \%$, as seen from the comparison with the measured like-sign spectra. The second source of background comes through incorrect associations of muons to non-muon tracks in the vertex tracker; it was estimated with an overlay Monte Carlo method (event mixing gives the same result). The $\omega$ and $\phi$ resonances are clearly seen, with $\sim 20 \mathrm{MeV}$ mass resolution.
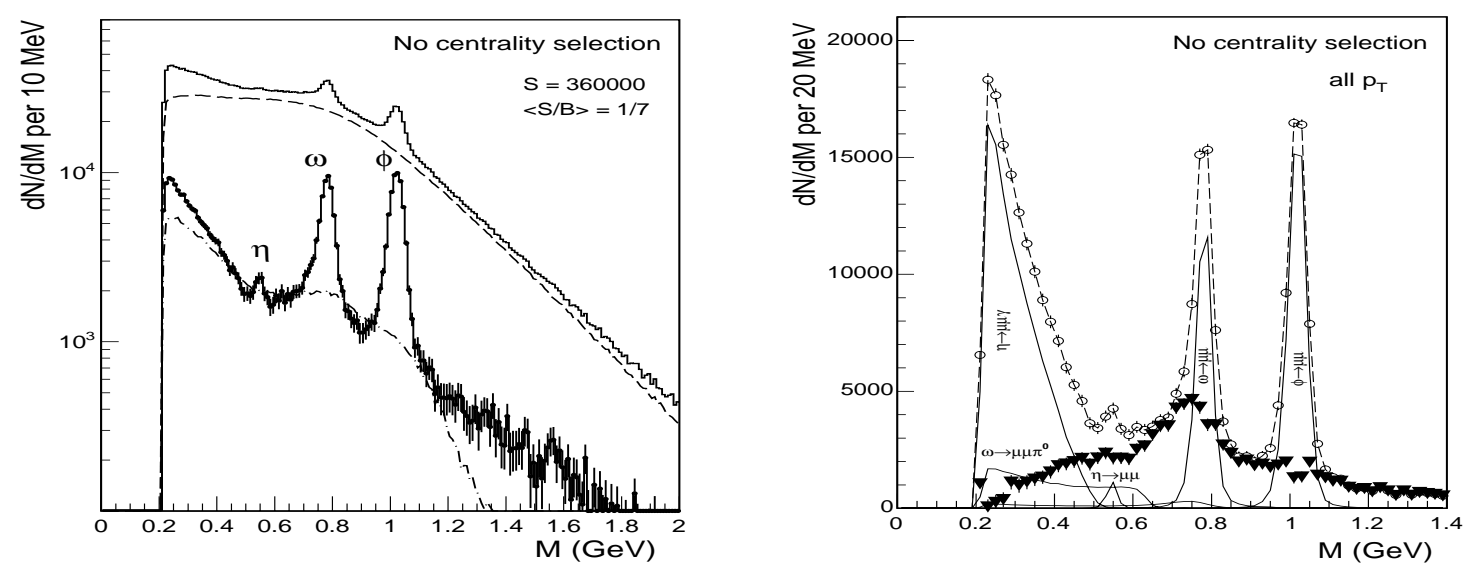

Figure 2: Left: Measured opposite-sign dimuon mass spectra (upper line), combinatorial background (dashed), fake matches (dashed-dotted) and extracted signal. Right: Illustration of the isolation procedure: the $\eta, \eta^{\prime}, \omega$ and $\phi$ contributions (solid lines) are subtracted from the signal spectrum (circles) to give the excess (triangles).

The expected $\eta, \eta^{\prime}, \rho, \omega$ and $\phi$ decays were simulated with the GENESIS [1, 5] generator, while the semi-muonic decays of D meson pairs were generated with PYTHIA. All generated muon pairs were placed inside real events and reconstructed as the real data. The events were binned in 4 classes of collision centrality, based on the charged-particle multiplicity distribution. The peripheral data, in $3 p_{\mathrm{T}}$ bins, can be well described with $\eta / \omega$ and $\phi / \omega$ cross-section ratios which are compatible with expectations from $\mathrm{p}$-A data and are, within $10 \%$, independent of $p_{\mathrm{T}}$ (a nice indication that the acceptance at low $p_{\mathrm{T}}$ is under control, even in the $\eta$ mass region). The $\rho / \omega$ ratio decreases with increasing $p_{\mathrm{T}}$, reaching 1.2 at high $p_{\mathrm{T}}$.

In the more central bins the data clearly shows an excess with respect to the expected sources. This excess, in each centrality bin, has been isolated by subtracting the cocktail sources (except for the $\rho$ ) from the data (see Fig. 2-right). The $\omega$ and $\phi$ yields are defined assuming a smooth 
underlying continuum. For the $\eta$, an upper limit is defined by "saturating" the measured data in the region close to $200 \mathrm{MeV}$; therefore, in this conservative approach, there is no excess at threshold, by construction. The excess mass spectra for the 4 multiplicity bins are shown in Fig. 3-left. For comparison purposes we show the unsubtracted cocktail $\rho$ (solid line), setting $\rho / \omega=1.2$, as seen at high $p_{\mathrm{T}}$ in the most peripheral bin. The charm contribution (dashed line) is fixed to $1 / 3$ at $M=1.2 \mathrm{GeV}$. The excess is centered at the position of the nominal $\rho$-pole and its yield increases with centrality, reaching a factor 4 with respect to the cocktail $\rho$ in the most central bin, when integrated in the range 200-900 MeV. The open data points in Fig. 3-right show the spectrum when the $\eta$-yield is decreased by $10 \%$. The systematical uncertainty on the excess yield, in the mass window surrounding the $\rho$ peak, is estimated to be around $25 \%$ in the two most central bins, and is dominated by the uncertainty on the background subtraction.
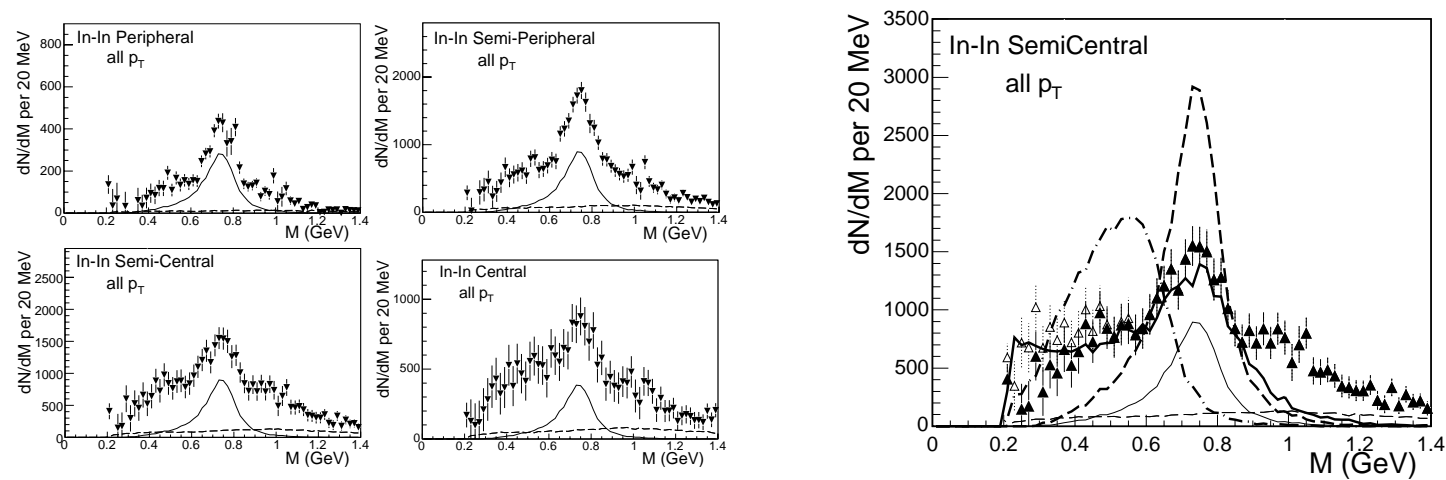

Figure 3: Excess dimuon mass spectra; the cocktail $\rho$ (thin solid) and the level of open charm (thin dashed) are shown for comparison. Left: Centrality dependence. Right: Comparison to model predictions [6], prepared for In-In at $\mathrm{d} N_{\mathrm{ch}} / \mathrm{d} \eta=140$. Unmodified $\rho$ (dashed), in-medium broadening $\rho$ (Rapp-Wambach, solid), in-medium dropping $\rho$ (related to Brown-Rho scaling, dashed-dotted).

Figure 3-right compares the excess to dimuon mass distributions generated according to theoretical models [6], tracked through the apparatus and reconstructed like the measured data. They are normalised to the excess in the mass range 200-900 MeV. The unmodified vacuum $\rho$ (dashed) and the dropping-mass scenario (dot-dashed) clearly fail to reproduce the measured excess, while the broadening scenario (solid) is in good agreement with the data, up to $900 \mathrm{MeV}$. More details can be found in [7]. We conclude that the shape of the excess broadens in In-In collisions, while no shift in mass is observed.

\section{References}

[1] G. Agakichiev et al. (CERES Coll.), Eur. Phys. J. C4 (1998) 231.

[2] G. Agakichiev et al. (CERES Coll.), Eur. Phys. J. C41 (2005) 475 and references therein.

[3] H.K. Wöhri et al. (NA60 Coll.), Eur. Phys. J. C43 (2005) 407.

[4] R. Shahoyan et al. (NA60 Coll.), these proceedings.

[5] S. Damjanovic, A. De Falco and H.K. Wöhri, "GENESIS, A hadron decay cocktail generator for the study of low mass dilepton production", NA60 Note 2005-1.

[6] R. Rapp and J. Wambach, Adv. Nucl. Phys. 25 (2000) 1; G.E. Brown and M. Rho, Phys. Rept. 363 (2002) 85; R. Rapp, private communication (2003).

[7] S. Damjanovic et al. (NA60 Coll.), Quark Matter 2005 proceedings; nucl-ex/0510044. 